\title{
Triple Diamond-Alpha integral and Hölder-type inequalities
}

Jing-Feng $\operatorname{Tian}^{1 *}$

\section{*Correspondence:}

tianjf@ncepu.edu.cn

${ }^{1}$ College of Science and

Technology, North China Electric

Power University, Baoding, P.R. China

\begin{abstract}
In this paper, we first introduce the definition of triple Diamond-Alpha integral for functions of three variables. Therefore, we present the Hölder and reverse Hölder inequalities for the triple Diamond-Alpha integral on time scales, and then we obtain some new generalizations of the Hölder and reverse Hölder inequalities for the triple Diamond-Alpha integral. Moreover, using the obtained results, we give a new generalization of the Minkowski inequality for the triple Diamond-Alpha integral on time scales.
\end{abstract}

MSC: $26 \mathrm{D} 15$

Keywords: Hölder's inequality; Minkowski's inequality; Triple Diamond-Alpha integral; Time scales

\section{Introduction}

To unify and generalize discrete and continuous analysis, in 1998, Hilger [1] introduced the theory of time scales. Since then, many researchers have studied various aspects of the theory and obtained a lot of interesting results on time scales [1-10]. The first purpose of this paper is to give the definition of the triple Diamond-Alpha integral (triple diamond- $\alpha$ integral or triple $\diamond_{\alpha}$-integral) for functions of three variables on time scales.

Let $u(x)$ and $v(x)$ be continuous real-valued functions on $[a, b]$, and let $\frac{1}{p}+\frac{1}{q}=1$.

(I) if $p>1$ and if $u(x) \geq 0, v(x) \geq 0$, then the classical Hölder inequality holds (see [11]):

$$
\int_{a}^{b} u(x) v(x) d x \leq\left(\int_{a}^{b} u^{p}(x) d x\right)^{\frac{1}{p}}\left(\int_{a}^{b} v^{q}(x) d x\right)^{\frac{1}{q}}
$$

(II) if $0<p<1$ and if $u(x)>0, v(x)>0$, then the following reverse Hölder inequality (e.g., see [12]) holds:

$$
\int_{a}^{b} u(x) v(x) d x \geq\left(\int_{a}^{b} u^{p}(x) d x\right)^{\frac{1}{p}}\left(\int_{a}^{b} v^{q}(x) d x\right)^{\frac{1}{q}}
$$

The classical Hölder and reverse Hölder inequalities play a very important role and have wide applications in different branches of modern mathematics. A large number of papers dealing with refinements, generalizations, and applications of the Hölder and reverse Hölder inequalities and their series analogues in different ares of mathematics have

(c) The Author(s) 2018. This article is distributed under the terms of the Creative Commons Attribution 4.0 International License (http://creativecommons.org/licenses/by/4.0/), which permits unrestricted use, distribution, and reproduction in any medium, provided you give appropriate credit to the original author(s) and the source, provide a link to the Creative Commons license, and indicate if changes were made. 
appeared. For example, Agahi et al. [13] gave generalizations of the Hölder and reverse Hölder inequalities for the pseudo-integral. Zhao et al. [14] found that the Hölder inequality for the pan-integral holds if the monotone measurer is subadditive. Tian [15-18] gave some new properties and refinements of the Hölder and reverse Hölder inequalities. For more detail, the reader may consult [19-25].

Among various extensions of (1) and (2), Agarwal, Bohner, and Peterson first presented the time scale versions of (1) via the Delta-integral ( $\Delta$-integral).

Theorem A Assume that $\mathbb{T}$ is a time scale, $a, b \in \mathbb{T}$, and $a<b$. If $p>1$ with $\frac{1}{p}+\frac{1}{q}=1$ and if $u, v \in C_{r d}([a, b], \mathbb{R})$, then

$$
\int_{a}^{b}|u(x) v(x)| \Delta x \leq\left(\int_{a}^{b}|u(x)|^{p} \Delta x\right)^{\frac{1}{p}}\left(\int_{a}^{b}|v(x)|^{q} \Delta x\right)^{\frac{1}{q}} .
$$

Later, in 2005, Wong et al. [26] gave the following Hölder-type inequalities via the Deltaintegral.

Theorem B Assume that $\mathbb{T}$ is a time scale, $a, b \in \mathbb{T}$, and $a<b$. Let $\frac{1}{p}+\frac{1}{q}=1$ and $u, v, \omega \in$ $C_{r d}([a, b], \mathbb{R})$. If $p>1$, then

$$
\int_{a}^{b}|\omega(x)||u(x) v(x)| \Delta x \leq\left(\int_{a}^{b}|\omega(x)||u(x)|^{p} \Delta x\right)^{\frac{1}{p}}\left(\int_{a}^{b}|\omega(x)||v(x)|^{q} \Delta x\right)^{\frac{1}{q}} .
$$

If $p<0$ or $q<0$, then inequality (4) is reversed.

In 2008, Özkan et al. [27] presented the following time scale versions of inequalities (1) and (2) via the Nabla-integral ( $\nabla$-integral) and Diamond-Alpha integral $\left(\diamond_{\alpha}\right.$-integral).

Theorem $\mathrm{C}$ Assume that $\mathbb{T}$ is a time scale, $a, b \in \mathbb{T}$, and $a<b$. Let $\frac{1}{p}+\frac{1}{q}=1$ and $u, v, \omega \in$ $C_{l d}([a, b], \mathbb{R})$. If $p>1$, then

$$
\int_{a}^{b}|\omega(x)||u(x) v(x)| \nabla x \leq\left(\int_{a}^{b}|\omega(x)||u(x)|^{p} \nabla x\right)^{\frac{1}{p}}\left(\int_{a}^{b}|\omega(x)||v(x)|^{q} \nabla x\right)^{\frac{1}{q}}
$$

If $p<0$ or $q<0$, then inequality (5) is reversed.

Theorem D Assume that $\mathbb{T}$ is a time scale, $a, b \in \mathbb{T}$, and $a<b$. Let $\frac{1}{p}+\frac{1}{q}=1$, and let $u, v, \omega:[a, b] \rightarrow \mathbb{R}$ be $\diamond_{\alpha}$-integrable functions. If $p>1$, then

$$
\int_{a}^{b}|\omega(x)||u(x) v(x)| \diamond_{\alpha} x \leq\left(\int_{a}^{b}|\omega(x)||u(x)|^{p} \diamond_{\alpha} x\right)^{\frac{1}{p}}\left(\int_{a}^{b}|\omega(x)||v(x)|^{q} \diamond_{\alpha} x\right)^{\frac{1}{q}} .
$$

If $p<0$ or $q<0$, then inequality (6) is reversed.

Remark 1.1 If $\alpha=0$ in Theorem C, then inequality (6) reduces to inequality (5). If $\alpha=1$ in Theorem $C$, then inequality (6) reduces to inequality (4).

The second purpose of this paper is to give the time scale versions of the Hölder and reverse Hölder inequalities for the triple Diamond-Alpha integral. Then we obtain some 
new generalizations of the Hölder and reverse Hölder inequalities for the triple DiamondAlpha integral on time scales. Moreover, using the obtained results, we present a new generalization of the Minkowski inequality for the triple Diamond-Alpha integral on time scales.

\section{Main results}

For details on time scales theory, the readers may consult [1,3-9] and the references therein. Now we give the definition of triple Diamond-Alpha integral for functions of three variables.

The triple Diamond-Alpha integral is defined as an iterated integral. Suppose that $\mathbb{T}$ is a time scale and $a_{i}, b_{i} \in \mathbb{T}$ with $a_{i}<b_{i}(i=1,2,3)$. Let $f\left(x_{1}, x_{2}, x_{3}\right)$ be a real-valued function on $\mathbb{T} \times \mathbb{T} \times \mathbb{T}$. Because we need the notation of partial derivatives with respect to variables $x_{i}$, we denote the time scale partial derivatives of $f\left(x_{1}, x_{2}, x_{3}\right)$ with respect to $x_{i}$ by $f_{\alpha}^{\lrcorner_{\alpha}^{i}}\left(x_{1}, x_{2}, x_{3}\right), i=1,2,3$. We now give the definition of these partial derivatives. Fixing $x_{2}, x_{3} \in \mathbb{T}$, the diamond- $\alpha$ derivative of a function

$$
\begin{aligned}
& \mathbb{T} \rightarrow \mathbb{R}, \\
& x_{1} \rightarrow f\left(x_{1}, x_{2}, x_{3}\right)
\end{aligned}
$$

is denoted by $f^{\diamond_{\alpha}^{1}}$. Next, fixing $x_{1}, x_{3} \in \mathbb{T}$, the diamond- $\alpha$ derivative of a function

$$
\begin{aligned}
& \mathbb{T} \rightarrow \mathbb{R}, \\
& x_{2} \rightarrow f\left(x_{1}, x_{2}, x_{3}\right)
\end{aligned}
$$

is denoted by $f^{\diamond_{\alpha}^{2}}$. Finally, fixing $x_{1}, x_{2} \in \mathbb{T}$, the diamond- $\alpha$ derivative of a function

$$
\begin{aligned}
& \mathbb{T} \rightarrow \mathbb{R}, \\
& x_{3} \rightarrow f\left(x_{1}, x_{2}, x_{3}\right)
\end{aligned}
$$

is denoted by $f^{\diamond_{\alpha}^{3}}$.

If a function $f$ has a $\diamond_{\alpha}^{1}$ antiderivative $F_{1}, F_{1}$ has a $\diamond_{\alpha}^{2}$ antiderivative $F_{2}$, and $F_{2}$ has a $\diamond_{\alpha}^{3}$ antiderivative $F_{3}$, that is, $F_{1}^{\diamond_{\alpha}^{1}}=f, F_{2}^{\diamond_{\alpha}^{2}}=F_{1}$, and $F_{3}^{\diamond_{\alpha}^{3}}=F_{2}$, then

$$
\begin{aligned}
\int_{a_{1}}^{b_{1}} & \int_{a_{2}}^{b_{2}} \int_{a_{3}}^{b_{3}} f\left(x_{1}, x_{2}, x_{3}\right) \diamond_{\alpha} x_{1} \diamond_{\alpha} x_{2} \diamond_{\alpha} x_{3} \\
:= & \int_{a_{2}}^{b_{2}} \int_{a_{3}}^{b_{3}}\left(F_{1}\left(b_{1}, x_{2}, x_{3}\right)-F_{1}\left(a_{1}, x_{2}, x_{3}\right)\right) \diamond_{\alpha} x_{2} \diamond_{\alpha} x_{3} \\
= & \int_{a_{3}}^{b_{3}}\left[F_{2}\left(b_{1}, b_{2}, x_{3}\right)-F_{2}\left(b_{1}, a_{2}, x_{3}\right)-\left(F_{2}\left(a_{1}, b_{2}, x_{3}\right)-F_{2}\left(a_{1}, a_{2}, x_{3}\right)\right)\right] \diamond_{\alpha} x_{3} \\
= & F_{3}\left(b_{1}, b_{2}, b_{3}\right)-F_{3}\left(b_{1}, b_{2}, a_{3}\right)-F_{3}\left(b_{1}, a_{2}, b_{3}\right)+F_{3}\left(b_{1}, a_{2}, a_{3}\right) \\
& -F_{3}\left(a_{1}, b_{2}, b_{3}\right)+F_{3}\left(a_{1}, b_{2}, a_{3}\right)+F_{3}\left(a_{1}, a_{2}, b_{3}\right)-F_{3}\left(a_{1}, a_{2}, a_{3}\right) .
\end{aligned}
$$

By this definition it is easy to obtain the following property for the triple Diamond-Alpha integral. 
Proposition 2.1 Suppose that $\mathbb{T}$ is a time scale, $a_{i}, b_{i} \in \mathbb{T}$ with $a_{i}<b_{i}(i=1,2,3)$, and $f\left(x_{1}, x_{2}, x_{3}\right)$ and $g\left(x_{1}, x_{2}, x_{3}\right)$ are $\diamond_{\alpha}$-integrable functions on $\left[a_{i}, b_{i}\right]_{\mathbb{T}}^{3}(i=1,2,3)$.

(i) If $f\left(x_{1}, x_{2}, x_{3}\right) \geq 0$ for all $x_{i} \in\left[a_{i}, b_{i}\right]_{\mathbb{T}}(i=1,2,3)$, then

$$
\int_{a_{1}}^{b_{1}} \int_{a_{2}}^{b_{2}} \int_{a_{3}}^{b_{3}} f\left(x_{1}, x_{2}, x_{3}\right) \diamond_{\alpha} x_{1} \diamond_{\alpha} x_{2} \diamond_{\alpha} x_{3} \geq 0
$$

(ii) If $f\left(x_{1}, x_{2}, x_{3}\right) \leq g\left(x_{1}, x_{2}, x_{3}\right)$ for all $x_{i} \in\left[a_{i}, b_{i}\right]_{\mathbb{T}}(i=1,2,3)$, then

$$
\begin{aligned}
& \int_{a_{1}}^{b_{1}} \int_{a_{2}}^{b_{2}} \int_{a_{3}}^{b_{3}} f\left(x_{1}, x_{2}, x_{3}\right) \diamond_{\alpha} x_{1} \diamond_{\alpha} x_{2} \diamond_{\alpha} x_{3} \\
& \quad \leq \int_{a_{1}}^{b_{1}} \int_{a_{2}}^{b_{2}} \int_{a_{3}}^{b_{3}} g\left(x_{1}, x_{2}, x_{3}\right) \diamond_{\alpha} x_{1} \diamond_{\alpha} x_{2} \diamond_{\alpha} x_{3} ;
\end{aligned}
$$

(iii) If $f\left(x_{1}, x_{2}, x_{3}\right) \geq 0$ for all $x_{i} \in\left[a_{i}, b_{i}\right]_{\mathbb{T}}(i=1,2,3)$, then $f\left(x_{1}, x_{2}, x_{3}\right)=0$ if and only if

$$
\int_{a_{1}}^{b_{1}} \int_{a_{2}}^{b_{2}} \int_{a_{3}}^{b_{3}} f\left(x_{1}, x_{2}, x_{3}\right) \diamond_{\alpha} x_{1} \diamond_{\alpha} x_{2} \diamond_{\alpha} x_{3}=0 .
$$

To prove the main results, we need the following lemmas.

Lemma 2.2 (Bernoulli's inequality; see [28]) If $x>0$ and $p>1$, then

$$
x^{p} \geq p x+1-p .
$$

Lemma 2.3 (Young inequality; see [11]) Let $a, b>0$.

(i) If $p, q>0$ with $\frac{1}{p}+\frac{1}{q}=1$, then

$$
a b \leq \frac{1}{p} a^{p}+\frac{1}{q} b^{q} .
$$

(ii) If $p>0, q<0$ with $\frac{1}{p}+\frac{1}{q}=1$, then

$$
a b \geq \frac{1}{p} a^{p}+\frac{1}{q} b^{q} .
$$

Lemma 2.4 (AG inequality; see [11]) Let $\alpha_{i}>0(i=1,2,3)$.

(i) If $1<\lambda_{1}, \lambda_{2}, \ldots, \lambda_{n}<\infty$ with $\sum_{i=1}^{n} \frac{1}{\lambda_{i}}=1$, then

$$
\prod_{i=1}^{n} \alpha_{i} \leq \sum_{i=1}^{n} \frac{\alpha_{i}^{\lambda_{i}}}{\lambda_{i}}
$$

(ii) If $\lambda_{1}>0, \lambda_{2}, \ldots, \lambda_{m}<0$ with $\sum_{i=1}^{m} \frac{1}{\lambda_{i}}=1$, then

$$
\prod_{i=1}^{m} \alpha_{i} \geq \sum_{i=1}^{m} \frac{\alpha_{i}^{\lambda_{i}}}{\lambda_{i}}
$$


Lemma 2.5 (Schlömilch's inequality for triple Diamond-Alpha integral) Suppose that $\mathbb{T}$ is a time scale, $a_{i}, b_{i} \in \mathbb{T}$ with $a_{i}<b_{i}(i=1,2,3)$, and $f\left(x_{1}, x_{2}, x_{3}\right), \omega\left(x_{1}, x_{2}, x_{3}\right):\left[a_{i}, b_{i}\right]_{\mathbb{T}}^{3} \rightarrow$ $[0,+\infty)$ are $\diamond_{\alpha}$-integrable functions with $\int_{a_{1}}^{b_{1}} \int_{a_{2}}^{b_{2}} \int_{a_{3}}^{b_{3}} \omega\left(x_{1}, x_{2}, x_{3}\right) \diamond_{\alpha} x_{1} \diamond_{\alpha} x_{2} \diamond_{\alpha} x_{3}>0$. Then, for $s>r>0$, we have

$$
\begin{aligned}
& \left(\frac{\int_{a_{1}}^{b_{1}} \int_{a_{2}}^{b_{2}} \int_{a_{3}}^{b_{3}} \omega\left(x_{1}, x_{2}, x_{3}\right) f^{s}\left(x_{1}, x_{2}, x_{3}\right) \diamond_{\alpha} x_{1} \diamond_{\alpha} x_{2} \diamond_{\alpha} x_{3}}{\int_{a_{1}}^{b_{1}} \int_{a_{2}}^{b_{2}} \int_{a_{3}}^{b_{3}} \omega\left(x_{1}, x_{2}, x_{3}\right) \diamond_{\alpha} x_{1} \diamond_{\alpha} x_{2} \diamond_{\alpha} x_{3}}\right)^{\frac{1}{s}} \\
& \geq\left(\frac{\int_{a_{1}}^{b_{1}} \int_{a_{2}}^{b_{2}} \int_{a_{3}}^{b_{3}} \omega\left(x_{1}, x_{2}, x_{3}\right) f^{r}\left(x_{1}, x_{2}, x_{3}\right) \diamond_{\alpha} x_{1} \diamond_{\alpha} x_{2} \diamond_{\alpha} x_{3}}{\int_{a_{1}}^{b_{1}} \int_{a_{2}}^{b_{2}} \int_{a_{3}}^{b_{3}} \omega\left(x_{1}, x_{2}, x_{3}\right) \diamond_{\alpha} x_{1} \diamond_{\alpha} x_{2} \diamond_{\alpha} x_{3}}\right)^{\frac{1}{r}} .
\end{aligned}
$$

Proof Without loss of generality, we may suppose that $\int_{a_{1}}^{b_{1}} \int_{a_{2}}^{b_{2}} \int_{a_{3}}^{b_{3}} h\left(x_{1}, x_{2}, x_{3}\right) \diamond_{\alpha} x_{1} \diamond_{\alpha} x_{2} \diamond_{\alpha}$ $x_{3}=1$. If $s>r>0$, then $\frac{s}{r}>1$. Therefore, by inequality (8) we have

$$
\begin{aligned}
& \int_{a_{1}}^{b_{1}} \int_{a_{2}}^{b_{2}} \int_{a_{3}}^{b_{3}}\left[\left(\frac{f\left(x_{1}, x_{2}, x_{3}\right)}{\int_{a_{1}}^{b_{1}} \int_{a_{2}}^{b_{2}} \int_{a_{3}}^{b_{3}} \omega\left(x_{1}, x_{2}, x_{3}\right) f\left(x_{1}, x_{2}, x_{3}\right) \diamond_{\alpha} x_{1} \diamond_{\alpha} x_{2} \diamond_{\alpha} x_{3}}\right)^{\frac{s}{r}}\right. \\
& \left.\quad \times \omega\left(x_{1}, x_{2}, x_{3}\right)\right] \diamond_{\alpha} x_{1} \diamond_{\alpha} x_{2} \diamond_{\alpha} x_{3} \\
& \geq \int_{a_{1}}^{b_{1}} \int_{a_{2}}^{b_{2}} \int_{a_{3}}^{b_{3}}\left[\left(\frac{\frac{s}{r} f\left(x_{1}, x_{2}, x_{3}\right)}{\int_{a}^{b} \int_{a}^{b} \cdots \int_{a}^{b} \omega\left(x_{1}, x_{2}, x_{3}\right) f\left(x_{1}, x_{2}, x_{3}\right) \diamond_{\alpha} x_{1} \diamond_{\alpha} x_{2} \diamond_{\alpha} x_{3}}+1-\frac{s}{r}\right)\right. \\
& \left.\quad \times \omega\left(x_{1}, x_{2}, x_{3}\right)\right] \diamond_{\alpha} x_{1} \diamond_{\alpha} x_{2} \diamond_{\alpha} x_{3}
\end{aligned}
$$

$=1$,

which implies

$$
\begin{aligned}
& \int_{a_{1}}^{b_{1}} \int_{a_{2}}^{b_{2}} \int_{a_{3}}^{b_{3}} \omega\left(x_{1}, x_{2}, x_{3}\right) f^{\frac{s}{r}}\left(x_{1}, x_{2}, x_{3}\right) \diamond_{\alpha} x_{1} \diamond_{\alpha} x_{2} \diamond_{\alpha} x_{3} \\
& \quad \geq\left(\int_{a_{1}}^{b_{1}} \int_{a_{2}}^{b_{2}} \int_{a_{3}}^{b_{3}} \omega\left(x_{1}, x_{2}, x_{3}\right) f\left(x_{1}, x_{2}, x_{3}\right) \diamond_{\alpha} x_{1} \diamond_{\alpha} x_{2} \diamond_{\alpha} x_{3}\right)^{\frac{s}{r}} .
\end{aligned}
$$

Replacing $f$ by $f^{r}$ in (13), we find

$$
\begin{aligned}
& \int_{a_{1}}^{b_{1}} \int_{a_{2}}^{b_{2}} \int_{a_{3}}^{b_{3}} \omega\left(x_{1}, x_{2}, x_{3}\right) f^{s}\left(x_{1}, x_{2}, x_{3}\right) \diamond_{\alpha} x_{1} \diamond_{\alpha} x_{2} \diamond_{\alpha} x_{3} \\
& \quad \geq\left(\int_{a_{1}}^{b_{1}} \int_{a_{2}}^{b_{2}} \int_{a_{3}}^{b_{3}} \omega\left(x_{1}, x_{2}, x_{3}\right) f^{r}\left(x_{1}, x_{2}, x_{3}\right) \diamond_{\alpha} x_{1} \diamond_{\alpha} x_{2} \diamond_{\alpha} x_{3}\right)^{\frac{s}{r}} .
\end{aligned}
$$

Then

$$
\begin{aligned}
& \left(\int_{a_{1}}^{b_{1}} \int_{a_{2}}^{b_{2}} \int_{a_{3}}^{b_{3}} \omega\left(x_{1}, x_{2}, x_{3}\right) f^{s}\left(x_{1}, x_{2}, x_{3}\right) \diamond_{\alpha} x_{1} \diamond_{\alpha} x_{2} \diamond_{\alpha} x_{3}\right)^{\frac{1}{s}} \\
& \quad \geq\left(\int_{a_{1}}^{b_{1}} \int_{a_{2}}^{b_{2}} \int_{a_{3}}^{b_{3}} \omega\left(x_{1}, x_{2}, x_{3}\right) f^{r}\left(x_{1}, x_{2}, x_{3}\right) \diamond_{\alpha} x_{1} \diamond_{\alpha} x_{2} \diamond_{\alpha} x_{3}\right)^{\frac{1}{r}} .
\end{aligned}
$$

Thus, the proof of Lemma 2.5 is completed. 
Now, we give the following Hölder and reverse Hölder inequalities for triple DiamondAlpha integral.

Theorem 2.6 Suppose that $\mathbb{T}$ is a time scale, $a_{i}, b_{i} \in \mathbb{T}$ with $a_{i}<b_{i}(i=1,2,3)$, and $f\left(x_{1}, x_{2}, x_{3}\right), g\left(x_{1}, x_{2}, x_{3}\right), \omega\left(x_{1}, x_{2}, x_{3}\right):\left[a_{i}, b_{i}\right]_{\mathbb{T}}^{3} \rightarrow \mathbb{R}$ are $\diamond_{\alpha}$-integrable functions.

(i) If $p, q>0$ are such that $\frac{1}{p}+\frac{1}{q}=1$, then

$$
\begin{aligned}
& \int_{a_{1}}^{b_{1}} \int_{a_{2}}^{b_{2}} \int_{a_{3}}^{b_{3}}\left|\omega\left(x_{1}, x_{2}, x_{3}\right) f\left(x_{1}, x_{2}, x_{3}\right) g\left(x_{1}, x_{2}, x_{3}\right)\right| \diamond_{\alpha} x_{1} \diamond_{\alpha} x_{2} \diamond_{\alpha} x_{3} \\
& \leq\left(\int_{a_{1}}^{b_{1}} \int_{a_{2}}^{b_{2}} \int_{a_{3}}^{b_{3}}\left|\omega\left(x_{1}, x_{2}, x_{3}\right)\right|\left|f\left(x_{1}, x_{2}, x_{3}\right)\right|^{p} \diamond_{\alpha} x_{1} \diamond_{\alpha} x_{2} \diamond_{\alpha} x_{3}\right)^{\frac{1}{p}} \\
& \quad \times\left(\int_{a_{1}}^{b_{1}} \int_{a_{2}}^{b_{2}} \int_{a_{3}}^{b_{3}}\left|\omega\left(x_{1}, x_{2}, x_{3}\right)\right|\left|g\left(x_{1}, x_{2}, x_{3}\right)\right|^{q} \diamond_{\alpha} x_{1} \diamond_{\alpha} x_{2} \diamond_{\alpha} x_{3}\right)^{\frac{1}{q}} .
\end{aligned}
$$

(ii) If $q<0$ is such that $\frac{1}{p}+\frac{1}{q}=1$, then

$$
\begin{aligned}
& \int_{a_{1}}^{b_{1}} \int_{a_{2}}^{b_{2}} \int_{a_{3}}^{b_{3}}\left|\omega\left(x_{1}, x_{2}, x_{3}\right) f\left(x_{1}, x_{2}, x_{3}\right) g\left(x_{1}, x_{2}, x_{3}\right)\right| \diamond_{\alpha} x_{1} \diamond_{\alpha} x_{2} \diamond_{\alpha} x_{3} \\
& \geq\left(\int_{a_{1}}^{b_{1}} \int_{a_{2}}^{b_{2}} \int_{a_{3}}^{b_{3}}\left|\omega\left(x_{1}, x_{2}, x_{3}\right)\right|\left|f\left(x_{1}, x_{2}, x_{3}\right)\right|^{p} \diamond_{\alpha} x_{1} \diamond_{\alpha} x_{2} \diamond_{\alpha} x_{3}\right)^{\frac{1}{p}} \\
& \quad \times\left(\int_{a_{1}}^{b_{1}} \int_{a_{2}}^{b_{2}} \int_{a_{3}}^{b_{3}}\left|\omega\left(x_{1}, x_{2}, x_{3}\right)\right|\left|g\left(x_{1}, x_{2}, x_{3}\right)\right|^{q} \diamond_{\alpha} x_{1} \diamond_{\alpha} x_{2} \diamond_{\alpha} x_{3}\right)^{\frac{1}{q}} .
\end{aligned}
$$

Proof Case (i): Let $p, q>0$ with $\frac{1}{p}+\frac{1}{q}=1$. Without loss of generality, we may suppose that

$$
\int_{a_{1}}^{b_{1}} \int_{a_{2}}^{b_{2}} \int_{a_{3}}^{b_{3}}\left|\omega\left(x_{1}, x_{2}, x_{3}\right)\right|\left|f\left(x_{1}, x_{2}, x_{3}\right)\right|^{p} \diamond_{\alpha} x_{1} \diamond_{\alpha} x_{2} \diamond_{\alpha} x_{3} \neq 0
$$

and

$$
\int_{a_{1}}^{b_{1}} \int_{a_{2}}^{b_{2}} \int_{a_{3}}^{b_{3}}\left|\omega\left(x_{1}, x_{2}, x_{3}\right)\right|\left|g\left(x_{1}, x_{2}, x_{3}\right)\right|^{q} \diamond_{\alpha} x_{1} \diamond_{\alpha} x_{2} \diamond_{\alpha} x_{3} \neq 0 .
$$

Let

$$
\mu=\frac{\left|\omega\left(x_{1}, x_{2}, x_{3}\right)\right|^{\frac{1}{p}}\left|f\left(x_{1}, x_{2}, x_{3}\right)\right|}{\left(\int_{a_{1}}^{b_{1}} \int_{a_{2}}^{b_{2}} \int_{a_{3}}^{b_{3}}\left|\omega\left(x_{1}, x_{2}, x_{3}\right)\right|\left|f\left(x_{1}, x_{2}, x_{3}\right)\right|^{p} \diamond_{\alpha} x_{1} \diamond_{\alpha} x_{2} \diamond_{\alpha} x_{3}\right)^{\frac{1}{p}}}
$$

and

$$
v=\frac{\left|\omega\left(x_{1}, x_{2}, x_{3}\right)\right|^{\frac{1}{q}}\left|g\left(x_{1}, x_{2}, x_{3}\right)\right|}{\left(\int_{a_{1}}^{b_{1}} \int_{a_{2}}^{b_{2}} \int_{a_{3}}^{b_{3}}\left|\omega\left(x_{1}, x_{2}, x_{3}\right)\right|\left|g\left(x_{1}, x_{2}, x_{3}\right)\right|^{q} \diamond_{\alpha} x_{1} \diamond_{\alpha} x_{2} \diamond_{\alpha} x_{3}\right)^{\frac{1}{q}}} .
$$


From the Young inequality (9) we get

$$
\begin{aligned}
\int_{a_{1}}^{b_{1}} & \int_{a_{2}}^{b_{2}} \int_{a_{3}}^{b_{3}} \mu \nu \diamond_{\alpha} x_{1} \diamond_{\alpha} x_{2} \diamond_{\alpha} x_{3} \\
\leq & \int_{a_{1}}^{b_{1}} \int_{a_{2}}^{b_{2}} \int_{a_{3}}^{b_{3}}\left(\frac{\mu^{p}}{p}+\frac{\nu^{q}}{q}\right) \diamond_{\alpha} x_{1} \diamond_{\alpha} x_{2} \diamond_{\alpha} x_{3} \\
= & \frac{1}{p} \int_{a_{1}}^{b_{1}} \int_{a_{2}}^{b_{2}} \int_{a_{3}}^{b_{3}}\left(\frac{\left|\omega\left(x_{1}, x_{2}, x_{3}\right)\right|}{\int_{a_{1}}^{b_{1}} \int_{a_{2}}^{b_{2}} \int_{a_{3}}^{b_{3}}\left|\omega\left(x_{1}, x_{2}, x_{3}\right)\right|\left|f\left(x_{1}, x_{2}, x_{3}\right)\right|^{p} \diamond_{\alpha} x_{1} \diamond_{\alpha} x_{2} \diamond_{\alpha} x_{3}}\right. \\
& \left.\times\left|f\left(x_{1}, x_{2}, x_{3}\right)\right|^{p}\right) \diamond_{\alpha} x_{1} \diamond_{\alpha} x_{2} \diamond_{\alpha} x_{3} \quad\left|\omega\left(x_{1}, x_{2}, x_{3}\right)\right| \\
& +\frac{1}{q} \int_{a_{1}}^{b_{1}} \int_{a_{2}}^{b_{2}} \int_{a_{3}}^{b_{3}}\left(\frac{\int_{a_{1}}^{b_{1}} \int_{a_{2}}^{b_{2}} \int_{a_{3}}^{b_{3}}\left|\omega\left(x_{1}, x_{2}, x_{3}\right)\right|\left|f\left(x_{1}, x_{2}, x_{3}\right)\right|^{q} \diamond_{\alpha} x_{1} \diamond_{\alpha} x_{2} \diamond_{\alpha} x_{3}}{{ }^{2}}\right. \\
& \left.\times\left|f\left(x_{1}, x_{2}, x_{3}\right)\right|^{q}\right) \diamond_{\alpha} x_{1} \diamond_{\alpha} x_{2} \diamond_{\alpha} x_{3} \\
= & \frac{1}{p}+\frac{1}{q}=1 .
\end{aligned}
$$

Therefore, we get the desired inequality (14).

Case (ii). Let $q<0$ with $\frac{1}{p}+\frac{1}{q}=1$. Write $\alpha=-\frac{p}{q}, \beta=\frac{1}{q}$. Then $\frac{1}{\alpha}+\frac{1}{\beta}=1$ with $\alpha, \beta>0$. Setting $f\left(x_{1}, x_{2}, x_{3}\right)=F\left(x_{1}, x_{2}, x_{3}\right)$ and $g\left(x_{1}, x_{2}, x_{3}\right)=G\left(x_{1}, x_{2}, x_{3}\right)$ in (14), we get

$$
\begin{aligned}
& \int_{a_{1}}^{b_{1}} \int_{a_{2}}^{b_{2}} \int_{a_{3}}^{b_{3}}\left|\omega\left(x_{1}, x_{2}, x_{3}\right) F\left(x_{1}, x_{2}, x_{3}\right) G\left(x_{1}, x_{2}, x_{3}\right)\right| \diamond_{\alpha} x_{1} \diamond_{\alpha} x_{2} \diamond_{\alpha} x_{3} \\
& \leq\left(\int_{a_{1}}^{b_{1}} \int_{a_{2}}^{b_{2}} \int_{a_{3}}^{b_{3}}\left|\omega\left(x_{1}, x_{2}, x_{3}\right)\right|\left|F\left(x_{1}, x_{2}, x_{3}\right)\right|^{\alpha} \diamond_{\alpha} x_{1} \diamond_{\alpha} x_{2} \diamond_{\alpha} x_{3}\right)^{\frac{1}{\alpha}} \\
& \quad \times\left(\int_{a_{1}}^{b_{1}} \int_{a_{2}}^{b_{2}} \int_{a_{3}}^{b_{3}}\left|\omega\left(x_{1}, x_{2}, x_{3}\right)\right|\left|G\left(x_{1}, x_{2}, x_{3}\right)\right|^{\beta} \diamond_{\alpha} x_{1} \diamond_{\alpha} x_{2} \diamond_{\alpha} x_{3}\right)^{\frac{1}{\beta}} .
\end{aligned}
$$

Putting $F\left(x_{1}, x_{2}, x_{3}\right)=f^{-q}\left(x_{1}, x_{2}, x_{3}\right)$ and $G\left(x_{1}, x_{2}, x_{3}\right)=f^{q}\left(x_{1}, x_{2}, x_{3}\right) g^{q}\left(x_{1}, x_{2}, x_{3}\right)$ in equality (16), we immediately obtain the desired inequality (15).

Next, we present the following generalizations of inequalities (14) and (15).

Theorem 2.7 Suppose that $\mathbb{T}$ is a time scale, $a_{i}, b_{i} \in \mathbb{T}$ with $a_{i}<b_{i}(i=1,2,3)$, and $f\left(x_{1}, x_{2}, x_{3}\right), g\left(x_{1}, x_{2}, x_{3}\right), \omega\left(x_{1}, x_{2}, x_{3}\right):\left[a_{i}, b_{i}\right]_{\mathbb{T}}^{3} \rightarrow \mathbb{R}$ are $\diamond_{\alpha}$-integrable functions.

(i) Let $\frac{1}{p}+\frac{1}{q}=\frac{1}{r}$ with $p, q, r \in \mathbb{R}-\{0\}, p>0$ and $q>0$ or $p>0, q<0$, and $r<0$. Then

$$
\begin{aligned}
& \left(\int_{a_{1}}^{b_{1}} \int_{a_{2}}^{b_{2}} \int_{a_{3}}^{b_{3}}\left|\omega\left(x_{1}, x_{2}, x_{3}\right) f^{r}\left(x_{1}, x_{2}, x_{3}\right) g^{r}\left(x_{1}, x_{2}, x_{3}\right)\right| \diamond_{\alpha} x_{1} \diamond_{\alpha} x_{2} \diamond_{\alpha} x_{3}\right)^{\frac{1}{r}} \\
& \leq\left(\int_{a_{1}}^{b_{1}} \int_{a_{2}}^{b_{2}} \int_{a_{3}}^{b_{3}}\left|\omega\left(x_{1}, x_{2}, x_{3}\right)\right|\left|f\left(x_{1}, x_{2}, x_{3}\right)\right|^{p} \diamond_{\alpha} x_{1} \diamond_{\alpha} x_{2} \diamond_{\alpha} x_{3}\right)^{\frac{1}{p}} \\
& \quad \times\left(\int_{a_{1}}^{b_{1}} \int_{a_{2}}^{b_{2}} \int_{a_{3}}^{b_{3}}\left|\omega\left(x_{1}, x_{2}, x_{3}\right)\right|\left|g\left(x_{1}, x_{2}, x_{3}\right)\right|^{q} \diamond_{\alpha} x_{1} \diamond_{\alpha} x_{2} \diamond_{\alpha} x_{3}\right)^{\frac{1}{q}}
\end{aligned}
$$


(ii) Let $\frac{1}{p}+\frac{1}{q}=\frac{1}{r}$ with $p, q, r \in \mathbb{R}-\{0\}, p>0, q<0$, and $r>0$ or $p<0$ and $q<0$. Then

$$
\begin{gathered}
\left(\int_{a_{1}}^{b_{1}} \int_{a_{2}}^{b_{2}} \int_{a_{3}}^{b_{3}}\left|\omega\left(x_{1}, x_{2}, x_{3}\right) f^{r}\left(x_{1}, x_{2}, x_{3}\right) g^{r}\left(x_{1}, x_{2}, x_{3}\right)\right| \diamond_{\alpha} x_{1} \diamond_{\alpha} x_{2} \diamond_{\alpha} x_{3}\right)^{\frac{1}{r}} \\
\geq\left(\int_{a_{1}}^{b_{1}} \int_{a_{2}}^{b_{2}} \int_{a_{3}}^{b_{3}}\left|\omega\left(x_{1}, x_{2}, x_{3}\right)\right|\left|f\left(x_{1}, x_{2}, x_{3}\right)\right|^{p} \diamond_{\alpha} x_{1} \diamond_{\alpha} x_{2} \diamond_{\alpha} x_{3}\right)^{\frac{1}{p}} \\
\quad \times\left(\int_{a_{1}}^{b_{1}} \int_{a_{2}}^{b_{2}} \int_{a_{3}}^{b_{3}}\left|\omega\left(x_{1}, x_{2}, x_{3}\right)\right|\left|g\left(x_{1}, x_{2}, x_{3}\right)\right|^{q} \diamond_{\alpha} x_{1} \diamond_{\alpha} x_{2} \diamond_{\alpha} x_{3}\right)^{\frac{1}{q}} .
\end{gathered}
$$

Proof (i) Case 1. When $p>0, q>0$, by $\frac{1}{p}+\frac{1}{q}=\frac{1}{r}$ we get

$$
\frac{p}{r}>1, \quad \frac{1}{p / r}+\frac{1}{q / r}=1
$$

Then, by (14) we find that

$$
\begin{aligned}
\int_{a_{1}}^{b_{1}} & \int_{a_{2}}^{b_{2}} \int_{a_{3}}^{b_{3}}\left|\omega\left(x_{1}, x_{2}, x_{3}\right) f^{r}\left(x_{1}, x_{2}, x_{3}\right) g^{r}\left(x_{1}, x_{2}, x_{3}\right)\right| \diamond_{\alpha} x_{1} \diamond_{\alpha} x_{2} \diamond_{\alpha} x_{3} \\
\leq & \left(\int_{a_{1}}^{b_{1}} \int_{a_{2}}^{b_{2}} \int_{a_{3}}^{b_{3}}\left|\omega\left(x_{1}, x_{2}, x_{3}\right) f^{r}\left(x_{1}, x_{2}, x_{3}\right)\right|^{\frac{p}{r}} \diamond_{\alpha} x_{1} \diamond_{\alpha} x_{2} \diamond_{\alpha} x_{3}\right)^{\frac{r}{p}} \\
& \times\left(\int_{a_{1}}^{b_{1}} \int_{a_{2}}^{b_{2}} \int_{a_{3}}^{b_{3}}\left|\omega\left(x_{1}, x_{2}, x_{3}\right) g^{r}\left(x_{1}, x_{2}, x_{3}\right)\right|^{\frac{q}{r}} \diamond_{\alpha} x_{1} \diamond_{\alpha} x_{2} \diamond_{\alpha} x_{3}\right)^{\frac{r}{q}} .
\end{aligned}
$$

Therefore

$$
\begin{gathered}
\left(\int_{a_{1}}^{b_{1}} \int_{a_{2}}^{b_{2}} \int_{a_{3}}^{b_{3}}\left|\omega\left(x_{1}, x_{2}, x_{3}\right) f^{r}\left(x_{1}, x_{2}, x_{3}\right) g^{r}\left(x_{1}, x_{2}, x_{3}\right)\right| \diamond_{\alpha} x_{1} \diamond_{\alpha} x_{2} \diamond_{\alpha} x_{3}\right)^{\frac{1}{r}} \\
\leq\left(\int_{a_{1}}^{b_{1}} \int_{a_{2}}^{b_{2}} \int_{a_{3}}^{b_{3}}\left|\omega\left(x_{1}, x_{2}, x_{3}\right) f^{r}\left(x_{1}, x_{2}, x_{3}\right)\right|^{\frac{p}{r}} \diamond_{\alpha} x_{1} \diamond_{\alpha} x_{2} \diamond_{\alpha} x_{3}\right)^{\frac{1}{p}} \\
\quad \times\left(\int_{a_{1}}^{b_{1}} \int_{a_{2}}^{b_{2}} \int_{a_{3}}^{b_{3}}\left|\omega\left(x_{1}, x_{2}, x_{3}\right) g^{r}\left(x_{1}, x_{2}, x_{3}\right)\right|^{\frac{q}{r}} \diamond_{\alpha} x_{1} \diamond_{\alpha} x_{2} \diamond_{\alpha} x_{3}\right)^{\frac{1}{q}}
\end{gathered}
$$

Case 2. When $p>0, q<0$, and $r<0$, by the same method as in Case 1 , we can obtain inequality (17).

(ii) Case I. When $p>0, q<0$, and $r>0$, we find from $\frac{1}{p}+\frac{1}{q}=\frac{1}{r}$ that

$$
\frac{r}{p}>1, \quad \frac{1}{r / p}+\frac{1}{-q / p}=\frac{p}{r}-\frac{p}{q}=p\left(\frac{1}{r}-\frac{1}{q}\right)=1 .
$$

Then, by inequality (14) we get

$$
\begin{gathered}
\int_{a_{1}}^{b_{1}} \int_{a_{2}}^{b_{2}} \int_{a_{3}}^{b_{3}}\left|\omega\left(x_{1}, x_{2}, x_{3}\right) f^{p}\left(x_{1}, x_{2}, x_{3}\right)\right| \diamond_{\alpha} x_{1} \diamond_{\alpha} x_{2} \diamond_{\alpha} x_{3} \\
\quad=\int_{a_{1}}^{b_{1}} \int_{a_{2}}^{b_{2}} \int_{a_{3}}^{b_{3}}\left(\left|\omega\left(x_{1}, x_{2}, x_{3}\right) f^{p}\left(x_{1}, x_{2}, x_{3}\right)\right|\right.
\end{gathered}
$$




$$
\begin{aligned}
& \left.\times\left|g^{p}\left(x_{1}, x_{2}, x_{3}\right) g^{-p}\left(x_{1}, x_{2}, x_{3}\right)\right|\right) \diamond_{\alpha} x_{1} \diamond_{\alpha} x_{2} \diamond_{\alpha} x_{3} \\
\leq & \left(\int_{a_{1}}^{b_{1}} \int_{a_{2}}^{b_{2}} \int_{a_{3}}^{b_{3}}\left|\omega\left(x_{1}, x_{2}, x_{3}\right)\right|\left|f^{p}\left(x_{1}, x_{2}, x_{3}\right) g^{p}\left(x_{1}, x_{2}, x_{3}\right)\right|^{\frac{r}{p}} \diamond_{\alpha} x_{1} \diamond_{\alpha} x_{2} \diamond_{\alpha} x_{3}\right)^{\frac{p}{r}} \\
& \times\left(\int_{a_{1}}^{b_{1}} \int_{a_{2}}^{b_{2}} \int_{a_{3}}^{b_{3}}\left|\omega\left(x_{1}, x_{2}, x_{3}\right)\right|\left|g^{-p}\left(x_{1}, x_{2}, x_{3}\right)\right|^{-\frac{q}{p}} \diamond_{\alpha} x_{1} \diamond_{\alpha} x_{2} \diamond_{\alpha} x_{3}\right)^{-\frac{p}{q}} \\
= & \left(\int_{a_{1}}^{b_{1}} \int_{a_{2}}^{b_{2}} \int_{a_{3}}^{b_{3}}\left|\omega\left(x_{1}, x_{2}, x_{3}\right)\right|\left|f^{r}\left(x_{1}, x_{2}, x_{3}\right) g^{r}\left(x_{1}, x_{2}, x_{3}\right)\right| \diamond_{\alpha} x_{1} \diamond_{\alpha} x_{2} \diamond_{\alpha} x_{3}\right)^{\frac{p}{r}} \\
& \times\left(\int_{a_{1}}^{b_{1}} \int_{a_{2}}^{b_{2}} \int_{a_{3}}^{b_{3}}\left|\omega\left(x_{1}, x_{2}, x_{3}\right)\right|\left|g^{q}\left(x_{1}, x_{2}, x_{3}\right)\right| \diamond_{\alpha} x_{1} \diamond_{\alpha} x_{2} \diamond_{\alpha} x_{3}\right)^{-\frac{p}{q}},
\end{aligned}
$$

which implies

$$
\begin{aligned}
& \left(\int_{a_{1}}^{b_{1}} \int_{a_{2}}^{b_{2}} \int_{a_{3}}^{b_{3}}\left|\omega\left(x_{1}, x_{2}, x_{3}\right) f^{p}\left(x_{1}, x_{2}, x_{3}\right)\right| \diamond_{\alpha} x_{1} \diamond_{\alpha} x_{2} \diamond_{\alpha} x_{3}\right)^{\frac{1}{p}} \\
& \quad \leq\left(\int_{a_{1}}^{b_{1}} \int_{a_{2}}^{b_{2}} \int_{a_{3}}^{b_{3}}\left|\omega\left(x_{1}, x_{2}, x_{3}\right)\right|\left|f^{r}\left(x_{1}, x_{2}, x_{3}\right) g^{r}\left(x_{1}, x_{2}, x_{3}\right)\right| \diamond_{\alpha} x_{1} \diamond_{\alpha} x_{2} \diamond_{\alpha} x_{3}\right)^{\frac{1}{r}} \\
& \quad \times\left(\int_{a_{1}}^{b_{1}} \int_{a_{2}}^{b_{2}} \int_{a_{3}}^{b_{3}}\left|\omega\left(x_{1}, x_{2}, x_{3}\right)\right|\left|g^{q}\left(x_{1}, x_{2}, x_{3}\right)\right| \diamond_{\alpha} x_{1} \diamond_{\alpha} x_{2} \diamond_{\alpha} x_{3}\right)^{-\frac{1}{q}} .
\end{aligned}
$$

Thus from inequality (19) we obtain

$$
\begin{gathered}
\left(\int_{a_{1}}^{b_{1}} \int_{a_{2}}^{b_{2}} \int_{a_{3}}^{b_{3}}\left|\omega\left(x_{1}, x_{2}, x_{3}\right) f^{r}\left(x_{1}, x_{2}, x_{3}\right) g^{r}\left(x_{1}, x_{2}, x_{3}\right)\right| \diamond_{\alpha} x_{1} \diamond_{\alpha} x_{2} \diamond_{\alpha} x_{3}\right)^{\frac{1}{r}} \\
\geq\left(\int_{a_{1}}^{b_{1}} \int_{a_{2}}^{b_{2}} \int_{a_{3}}^{b_{3}}\left|\omega\left(x_{1}, x_{2}, x_{3}\right)\right|\left|f\left(x_{1}, x_{2}, x_{3}\right)\right|^{p} \diamond_{\alpha} x_{1} \diamond_{\alpha} x_{2} \diamond_{\alpha} x_{3}\right)^{\frac{1}{p}} \\
\quad \times\left(\int_{a_{1}}^{b_{1}} \int_{a_{2}}^{b_{2}} \int_{a_{3}}^{b_{3}}\left|\omega\left(x_{1}, x_{2}, x_{3}\right)\right|\left|g\left(x_{1}, x_{2}, x_{3}\right)\right|^{q} \diamond_{\alpha} x_{1} \diamond_{\alpha} x_{2} \diamond_{\alpha} x_{3}\right)^{\frac{1}{q}} .
\end{gathered}
$$

Case II. When $p<0$ and $q<0$, by the same method as in Case I, we can obtain the desired inequality (18). The proof of Theorem 2.7 is completed.

We present another generalization of inequality (14).

Theorem 2.8 Suppose that $\mathbb{T}$ is a time scale, $a_{i}, b_{i} \in \mathbb{T}$ with $a_{i}<b_{i}(i=1,2,3)$, and that $f\left(x_{1}, x_{2}, x_{3}\right), g\left(x_{1}, x_{2}, x_{3}\right), \omega\left(x_{1}, x_{2}, x_{3}\right):\left[a_{i}, b_{i}\right]_{\mathbb{T}}^{3} \rightarrow \mathbb{R}$ are $\diamond_{\alpha}$-integrable functions. If $p>0$, $q>0$ are such that $0<\frac{1}{p}+\frac{1}{q}<1$, then

$$
\begin{aligned}
\int_{a_{1}}^{b_{1}} \int_{a_{2}}^{b_{2}} \int_{a_{3}}^{b_{3}}\left|\omega\left(x_{1}, x_{2}, x_{3}\right) f\left(x_{1}, x_{2}, x_{3}\right) g\left(x_{1}, x_{2}, x_{3}\right)\right| \diamond_{\alpha} x_{1} \diamond_{\alpha} x_{2} \diamond_{\alpha} x_{3} \\
\leq\left(\int_{a_{1}}^{b_{1}} \int_{a_{2}}^{b_{2}} \int_{a_{3}}^{b_{3}}\left|\omega\left(x_{1}, x_{2}, x_{3}\right)\right|\left|f\left(x_{1}, x_{2}, x_{3}\right)\right|^{p} \diamond_{\alpha} x_{1} \diamond_{\alpha} x_{2} \diamond_{\alpha} x_{3}\right)^{\frac{1}{p}} \\
\quad \times\left(\int_{a_{1}}^{b_{1}} \int_{a_{2}}^{b_{2}} \int_{a_{3}}^{b_{3}}\left|\omega\left(x_{1}, x_{2}, x_{3}\right)\right|\left|g\left(x_{1}, x_{2}, x_{3}\right)\right|^{q} \diamond_{\alpha} x_{1} \diamond_{\alpha} x_{2} \diamond_{\alpha} x_{3}\right)^{\frac{1}{q}} .
\end{aligned}
$$


Proof Denote $\gamma:=\frac{1}{p}+\frac{1}{q}, \zeta=\gamma p, \delta=q \gamma$. Then $\zeta>1, \delta>1$, and $\frac{1}{\zeta}+\frac{1}{\delta}=1$. Hence from Lemma 2.5 with $\zeta<p, \delta<q$ and inequality (14) we find that

$$
\begin{aligned}
\int_{a_{1}}^{b_{1}} & \int_{a_{2}}^{b_{2}} \int_{a_{3}}^{b_{3}}\left|\omega\left(x_{1}, x_{2}, x_{3}\right) f\left(x_{1}, x_{2}, x_{3}\right) g\left(x_{1}, x_{2}, x_{3}\right)\right| \diamond_{\alpha} x_{1} \diamond_{\alpha} x_{2} \diamond_{\alpha} x_{3} \\
\leq & \left(\int_{a_{1}}^{b_{1}} \int_{a_{2}}^{b_{2}} \int_{a_{3}}^{b_{3}}\left|\omega\left(x_{1}, x_{2}, x_{3}\right)\right|\left|f\left(x_{1}, x_{2}, x_{3}\right)\right|^{\zeta} \diamond_{\alpha} x_{1} \diamond_{\alpha} x_{2} \diamond_{\alpha} x_{3}\right)^{\frac{1}{\zeta}} \\
& \times\left(\int_{a_{1}}^{b_{1}} \int_{a_{2}}^{b_{2}} \int_{a_{3}}^{b_{3}}\left|\omega\left(x_{1}, x_{2}, x_{3}\right)\right|\left|g\left(x_{1}, x_{2}, x_{3}\right)\right|^{\delta} \diamond_{\alpha} x_{1} \diamond_{\alpha} x_{2} \diamond_{\alpha} x_{3}\right)^{\frac{1}{\delta}} \\
\leq & \left(\int_{a_{1}}^{b_{1}} \int_{a_{2}}^{b_{2}} \int_{a_{3}}^{b_{3}}\left|\omega\left(x_{1}, x_{2}, x_{3}\right)\right|\left|f\left(x_{1}, x_{2}, x_{3}\right)\right|^{p} \diamond_{\alpha} x_{1} \diamond_{\alpha} x_{2} \diamond_{\alpha} x_{3}\right)^{\frac{1}{p}} \\
& \times\left(\int_{a_{1}}^{b_{1}} \int_{a_{2}}^{b_{2}} \int_{a_{3}}^{b_{3}}\left|\omega\left(x_{1}, x_{2}, x_{3}\right)\right|\left|g\left(x_{1}, x_{2}, x_{3}\right)\right|^{q} \diamond_{\alpha} x_{1} \diamond_{\alpha} x_{2} \cdots \diamond_{\alpha} x_{n}\right)^{\frac{1}{q}}
\end{aligned}
$$

The proof of Theorem 2.8 is completed.

Theorem 2.9 Suppose that $\mathbb{T}$ is a time scale, $a_{i}, b_{i} \in \mathbb{T}$ with $a_{i}<b_{i}(i=1,2,3)$, and that $f_{i}\left(x_{1}, x_{2}, x_{3}\right)(i=1,2, \ldots, m), \omega\left(x_{1}, x_{2}, x_{3}\right):\left[a_{i}, b_{i}\right]_{\mathbb{T}}^{3} \rightarrow \mathbb{R}$ are $\diamond_{\alpha}$-integrable functions.

(I) Let $1<\lambda_{1}, \lambda_{2}, \ldots, \lambda_{m}<\infty$ such that $\sum_{i=1}^{m} \frac{1}{\lambda_{i}}=1$. Then

$$
\begin{aligned}
& \int_{a_{1}}^{b_{1}} \int_{a_{2}}^{b_{2}} \int_{a_{3}}^{b_{3}}\left|\omega\left(x_{1}, x_{2}, x_{3}\right)\left(\prod_{i=1}^{m} f_{i}\left(x_{1}, x_{2}, x_{3}\right)\right)\right| \diamond_{\alpha} x_{1} \diamond_{\alpha} x_{2} \diamond_{\alpha} x_{3} \\
& \leq \prod_{i=1}^{m}\left(\int_{a_{1}}^{b_{1}} \int_{a_{2}}^{b_{2}} \int_{a_{3}}^{b_{3}}\left|\omega\left(x_{1}, x_{2}, x_{3}\right)\right|\left|f_{i}\left(x_{1}, x_{2}, x_{3}\right)\right|^{\lambda_{i}} \diamond_{\alpha} x_{1} \diamond_{\alpha} x_{2} \diamond_{\alpha} x_{3}\right)^{\frac{1}{\lambda_{i}}}
\end{aligned}
$$

(II) Let $\lambda_{1}>0, \lambda_{2}, \ldots, \lambda_{m}<0$ be such that $\sum_{i=1}^{m} \frac{1}{\lambda_{i}}=1$. Then inequality (21) is reversed.

Proof Case (I). Without loss of generality, we may suppose that

$$
\prod_{i=1}^{m}\left(\int_{a_{1}}^{b_{1}} \int_{a_{2}}^{b_{2}} \int_{a_{3}}^{b_{3}}\left|\omega\left(x_{1}, x_{2}, x_{3}\right)\right|\left|f_{i}\left(x_{1}, x_{2}, x_{3}\right)\right|^{\lambda_{i}} \diamond_{\alpha} x_{1} \diamond_{\alpha} x_{2} \diamond_{\alpha} x_{3}\right)^{\frac{1}{\lambda_{i}}} \neq 0 .
$$

Write

$$
\alpha_{i}=\frac{\left|\omega\left(x_{1}, x_{2}, x_{3}\right)\right|^{\frac{1}{\lambda_{i}}}\left|f_{i}\left(x_{1}, x_{2}, x_{3}\right)\right|}{\left(\int_{a_{1}}^{b_{1}} \int_{a_{2}}^{b_{2}} \int_{a_{3}}^{b_{3}}\left|\omega\left(x_{1}, x_{2}, x_{3}\right)\right|\left|f_{i}\left(x_{1}, x_{2}, x_{3}\right)\right|^{\lambda_{i}} \diamond_{\alpha} x_{1} \diamond_{\alpha} x_{2} \diamond_{\alpha} x_{3}\right)^{\frac{1}{\lambda_{i}}}}
$$

for $i=1,2, \ldots, m$.

From $A G$ inequality (11) we have

$$
\begin{aligned}
& \int_{a_{1}}^{b_{1}} \int_{a_{2}}^{b_{2}} \int_{a_{3}}^{b_{3}} \prod_{i=1}^{m} \alpha_{i} \diamond_{\alpha} x_{1} \diamond_{\alpha} x_{2} \diamond_{\alpha} x_{3} \\
& \leq \int_{a_{1}}^{b_{1}} \int_{a_{2}}^{b_{2}} \int_{a_{3}}^{b_{3}}\left(\sum_{i=1}^{m} \frac{\alpha_{i}^{\lambda_{i}}}{\lambda_{i}}\right) \diamond_{\alpha} x_{1} \diamond_{\alpha} x_{2} \diamond_{\alpha} x_{3}
\end{aligned}
$$




$$
\begin{aligned}
& =\sum_{i=1}^{m} \frac{1}{\lambda_{i}} \int_{a_{1}}^{b_{1}} \int_{a_{2}}^{b_{2}} \int_{a_{3}}^{b_{3}}\left(\frac{\left|\omega\left(x_{1}, x_{2}, x_{3}\right)\right|}{\int_{a_{1}}^{b_{1}} \int_{a_{2}}^{b_{2}} \int_{a_{3}}^{b_{3}}\left|\omega\left(x_{1}, x_{2}, x_{3}\right)\right|\left|f_{i}\left(x_{1}, x_{2}, x_{3}\right)\right|^{\lambda_{i} \diamond_{\alpha} x_{1} \diamond_{\alpha} x_{2} \diamond_{\alpha} x_{3}}}\right. \\
& \left.\quad \times\left|f_{i}\left(x_{1}, x_{2}, x_{3}\right)\right|^{\lambda_{i}}\right) \diamond_{\alpha} x_{1} \diamond_{\alpha} x_{2} \diamond_{\alpha} x_{3} \\
& =\sum_{i=1}^{m} \frac{1}{\lambda_{i}}=1 .
\end{aligned}
$$

Therefore, we get the desired inequality (21).

Case (II). By the same method as in Case (I) and using the reversed inequality (11), we can obtain the desired result.

Theorem 2.10 Suppose that $\mathbb{T}$ is a time scale, $a_{i}, b_{i} \in \mathbb{T}$ with $a_{i}<b_{i}(i=1,2,3)$, and that $f_{i}\left(x_{1}, x_{2}, x_{3}\right), \omega\left(x_{1}, x_{2}, x_{3}\right):\left[a_{i}, b_{i}\right]_{\mathbb{T}}^{3} \rightarrow[0,+\infty)(i=1,2, \ldots, m)$ are $\diamond_{\alpha}$-integrable functions with $\int_{a_{1}}^{b_{1}} \int_{a_{2}}^{b_{2}} \int_{a_{3}}^{b_{3}} \omega\left(x_{1}, x_{2}, x_{3}\right) \diamond_{\alpha} x_{1} \diamond_{\alpha} x_{2} \diamond_{\alpha} x_{3}=1$. Let $0<\lambda_{1}, \lambda_{2}, \ldots, \lambda_{m}<1$ be such that $\lambda_{1}+\lambda_{2}+\cdots+\lambda_{m}=k<1$. Then

$$
\begin{aligned}
& \int_{a_{1}}^{b_{1}} \int_{a_{2}}^{b_{2}} \int_{a_{3}}^{b_{3}} \omega\left(x_{1}, x_{2}, x_{3}\right)\left(\prod_{i=1}^{m} f_{i}^{\lambda_{i}}\left(x_{1}, x_{2}, x_{3}\right)\right) \diamond_{\alpha} x_{1} \diamond_{\alpha} x_{2} \diamond_{\alpha} x_{3} \\
& \quad \leq \prod_{i=1}^{m}\left(\int_{a_{1}}^{b_{1}} \int_{a_{2}}^{b_{2}} \int_{a_{3}}^{b_{3}} \omega\left(x_{1}, x_{2}, x_{3}\right) f_{i}\left(x_{1}, x_{2}, x_{3}\right) \diamond_{\alpha} x_{1} \diamond_{\alpha} x_{2} \diamond_{\alpha} x_{3}\right)^{\lambda_{i}} .
\end{aligned}
$$

Proof Denote $\xi_{i}=\frac{\lambda_{i}}{k}(i=1,2, \ldots, m)$. Then

$$
\xi_{1}+\xi_{2}+\cdots+\xi_{m}=1
$$

Write

$$
\psi_{i}\left(x_{1}, x_{2}, x_{3}\right)=f_{i}^{k}\left(x_{1}, x_{2}, x_{3}\right) \quad(i=1,2, \ldots, m) .
$$

From Theorem 2.9 and Lemma 2.5 we have

$$
\begin{aligned}
& \int_{a_{1}}^{b_{1}} \int_{a_{2}}^{b_{2}} \int_{a_{3}}^{b_{3}} \omega\left(x_{1}, x_{2}, x_{3}\right) \prod_{i=1}^{m} f_{i}^{\lambda_{i}}\left(x_{1}, x_{2}, x_{3}\right) \diamond_{\alpha} x_{1} \diamond_{\alpha} x_{2} \diamond_{\alpha} x_{3} \\
& \quad=\int_{a_{1}}^{b_{1}} \int_{a_{2}}^{b_{2}} \int_{a_{3}}^{b_{3}} \omega\left(x_{1}, x_{2}, x_{3}\right) \prod_{i=1}^{m} \psi_{i}^{\xi_{i}}\left(x_{1}, x_{2}, x_{3}\right) \diamond_{\alpha} x_{1} \diamond_{\alpha} x_{2} \diamond_{\alpha} x_{3} \\
& \quad \leq \prod_{i=1}^{m}\left(\int_{a_{1}}^{b_{1}} \int_{a_{2}}^{b_{2}} \int_{a_{3}}^{b_{3}} \omega\left(x_{1}, x_{2}, x_{3}\right) \psi_{i}\left(x_{1}, x_{2}, x_{3}\right) \diamond_{\alpha} x_{1} \diamond_{\alpha} x_{2} \diamond_{\alpha} x_{3}\right)^{\xi_{i}} \\
& \quad=\prod_{i=1}^{m}\left(\int_{a_{1}}^{b_{1}} \int_{a_{2}}^{b_{2}} \int_{a_{3}}^{b_{3}} \omega\left(x_{1}, x_{2}, x_{3}\right) f_{i}^{k}\left(x_{1}, x_{2}, x_{3}\right) \diamond_{\alpha} x_{1} \diamond_{\alpha} x_{2} \diamond_{\alpha} x_{3}\right)^{\frac{\lambda_{i}}{k}} \\
& \quad \leq \prod_{i=1}^{m}\left(\int_{a_{1}}^{b_{1}} \int_{a_{2}}^{b_{2}} \int_{a_{3}}^{b_{3}} \omega\left(x_{1}, x_{2}, x_{3}\right) f_{i}\left(x_{1}, x_{2}, x_{3}\right) \diamond_{\alpha} x_{1} \diamond_{\alpha} x_{2} \diamond_{\alpha} x_{3}\right)^{\lambda_{i}}
\end{aligned}
$$

for $k<1$. Therefore the proof of Theorem 2.10 is completed. 
Let $f_{i}^{\lambda_{i}}\left(x_{1}, x_{2}, x_{3}\right)=\mu_{i}\left(x_{1}, x_{2}, x_{3}\right)$, that is, $f_{i}\left(x_{1}, x_{2}, x_{3}\right)=\mu_{i}^{\frac{1}{\lambda_{i}}}\left(x_{1}, x_{2}, x_{3}\right)$ for $i=1,2, \ldots, m$. Then from Theorem 2.10 we get the following Hölder-type inequality.

Corollary 2.11 Suppose that $\mathbb{T}$ is a time scale, $a_{i}, b_{i} \in \mathbb{T}$ with $a_{i}<b_{i}(i=1,2,3)$, and that $g_{i}\left(x_{1}, x_{2}, x_{3}\right), \omega\left(x_{1}, x_{2}, x_{3}\right):\left[a_{i}, b_{i}\right]_{\mathbb{T}}^{3} \rightarrow[0,+\infty)(i=1,2, \ldots, m)$ are $\diamond_{\alpha}$-integrable functions with $\int_{a_{1}}^{b_{1}} \int_{a_{2}}^{b_{2}} \int_{a_{3}}^{b_{3}} \omega\left(x_{1}, x_{2}, x_{3}\right) \diamond_{\alpha} x_{1} \diamond_{\alpha} x_{2} \diamond_{\alpha} x_{3}=1$. Let $0<\lambda_{1}, \lambda_{2}, \ldots, \lambda_{m}<1$ be such that $\lambda_{1}+\lambda_{2}+\cdots+\lambda_{m}=k<1$. Then

$$
\begin{aligned}
& \int_{a_{1}}^{b_{1}} \int_{a_{2}}^{b_{2}} \int_{a_{3}}^{b_{3}} \omega\left(x_{1}, x_{2}, x_{3}\right)\left(\prod_{i=1}^{m} g_{i}\left(x_{1}, x_{2}, x_{3}\right)\right) \diamond_{\alpha} x_{1} \diamond_{\alpha} x_{2} \diamond_{\alpha} x_{3} \\
& \quad \leq \prod_{i=1}^{m}\left(\int_{a_{1}}^{b_{1}} \int_{a_{2}}^{b_{2}} \int_{a_{3}}^{b_{3}} \omega\left(x_{1}, x_{2}, x_{3}\right) g_{i}^{\frac{1}{\lambda_{i}}}\left(x_{1}, x_{2}, x_{3}\right) \diamond_{\alpha} x_{1} \diamond_{\alpha} x_{2} \diamond_{\alpha} x_{3}\right)^{\lambda_{i}} .
\end{aligned}
$$

\section{Application}

In this section, using the obtained results, we give the following generalization of the Minkowski inequality for the triple Diamond-Alpha integral on time scales.

Theorem 3.1 Suppose that $\mathbb{T}$ is a time scale, $a_{i}, b_{i} \in \mathbb{T}$ with $a_{i}<b_{i}(i=1,2,3)$, and that $f_{i}\left(x_{1}, x_{2}, x_{3}\right)(i=1,2, \ldots, m)$ and $\omega\left(x_{1}, x_{2}, x_{3}\right):\left[a_{i}, b_{i}\right]_{\mathbb{T}}^{3} \rightarrow \mathbb{R}$ are $\diamond_{\alpha}$-integrable functions.

(I) If $p>1$, then

$$
\begin{aligned}
& {\left[\int_{a_{1}}^{b_{1}} \int_{a_{2}}^{b_{2}} \int_{a_{3}}^{b_{3}}\left|\omega\left(x_{1}, x_{2}, x_{3}\right)\left(\sum_{i=1}^{m} f_{i}\left(x_{1}, x_{2}, x_{3}\right)\right)^{p}\right| \diamond_{\alpha} x_{1} \diamond_{\alpha} x_{2} \diamond_{\alpha} x_{3}\right]^{\frac{1}{p}}} \\
& \quad \leq \sum_{i=1}^{m}\left(\int_{a_{1}}^{b_{1}} \int_{a_{2}}^{b_{2}} \int_{a_{3}}^{b_{3}}\left|\omega\left(x_{1}, x_{2}, x_{3}\right)\right|\left|f_{i}\left(x_{1}, x_{2}, x_{3}\right)\right|^{p} \diamond_{\alpha} x_{1} \diamond_{\alpha} x_{2} \diamond_{\alpha} x_{3}\right)^{\frac{1}{p}}
\end{aligned}
$$

(II) If $0<p<1$, then inequality (25) is reversed.

Proof We prove only case (I). Write $\Phi\left(x_{1}, x_{2}, x_{3}\right)=\sum_{i=1}^{m} f_{i}\left(x_{1}, x_{2}, x_{3}\right)$. Without loss of generality, we may assume that $\int_{a_{1}}^{b_{1}} \int_{a_{2}}^{b_{2}} \int_{a_{3}}^{b_{3}} \omega\left(x_{1}, x_{2}, x_{3}\right) \Phi^{p}\left(x_{1}, x_{2}, x_{3}\right) \diamond_{\alpha} x_{1} \diamond_{\alpha} x_{2} \diamond_{\alpha} x_{3} \neq 0$. From inequality (14) for $p, q>0$ and $\frac{1}{p}+\frac{1}{q}=1$ we have

$$
\begin{aligned}
\int_{a_{1}}^{b_{1}} & \int_{a_{2}}^{b_{2}} \int_{a_{3}}^{b_{3}}\left|f_{i}\left(x_{1}, x_{2}, x_{3}\right) \omega\left(x_{1}, x_{2}, x_{3}\right) \Phi^{p-1}\left(x_{1}, x_{2}, x_{3}\right)\right| \diamond_{\alpha} x_{1} \diamond_{\alpha} x_{2} \diamond_{\alpha} x_{3} \\
\leq & \left(\int_{a_{1}}^{b_{1}} \int_{a_{2}}^{b_{2}} \int_{a_{3}}^{b_{3}}\left|\omega\left(x_{1}, x_{2}, x_{3}\right)\right|\left|f_{i}\left(x_{1}, x_{2}, x_{3}\right)\right|^{p} \diamond_{\alpha} x_{1} \diamond_{\alpha} x_{2} \diamond_{\alpha} x_{3}\right)^{\frac{1}{p}} \\
& \times\left(\int_{a_{1}}^{b_{1}} \int_{a_{2}}^{b_{2}} \int_{a_{3}}^{b_{3}}\left|\omega\left(x_{1}, x_{2}, x_{3}\right)\right|\left|\Phi\left(x_{1}, x_{2}, x_{3}\right)\right|^{(p-1) q} \diamond_{\alpha} x_{1} \diamond_{\alpha} x_{2} \diamond_{\alpha} x_{3}\right)^{\frac{1}{q}} \\
= & \left(\int_{a_{1}}^{b_{1}} \int_{a_{2}}^{b_{2}} \int_{a_{3}}^{b_{3}}\left|\omega\left(x_{1}, x_{2}, x_{3}\right)\right|\left|f_{i}\left(x_{1}, x_{2}, x_{3}\right)\right|^{p} \diamond_{\alpha} x_{1} \diamond_{\alpha} x_{2} \diamond_{\alpha} x_{3}\right)^{\frac{1}{p}} \\
& \times\left(\int_{a_{1}}^{b_{1}} \int_{a_{2}}^{b_{2}} \int_{a_{3}}^{b_{3}}\left|\omega\left(x_{1}, x_{2}, x_{3}\right)\right|\left|\Phi\left(x_{1}, x_{2}, x_{3}\right)\right|^{p} \diamond_{\alpha} x_{1} \diamond_{\alpha} x_{2} \diamond_{\alpha} x_{3}\right)^{\frac{1}{q}} .
\end{aligned}
$$


Therefore we get

$$
\begin{aligned}
& \int_{a_{1}}^{b_{1}} \int_{a_{2}}^{b_{2}} \int_{a_{3}}^{b_{3}}\left|\omega\left(x_{1}, x_{2}, x_{3}\right) \Phi^{p}\left(x_{1}, x_{2}, x_{3}\right)\right| \diamond_{\alpha} x_{1} \diamond_{\alpha} x_{2} \diamond_{\alpha} x_{3} \\
&=\int_{a_{1}}^{b_{1}} \int_{a_{2}}^{b_{2}} \int_{a_{3}}^{b_{3}}\left|\omega\left(x_{1}, x_{2}, x_{3}\right) \Phi\left(x_{1}, x_{2}, x_{3}\right) \Phi^{p-1}\left(x_{1}, x_{2}, x_{3}\right)\right| \diamond_{\alpha} x_{1} \diamond_{\alpha} x_{2} \diamond_{\alpha} x_{3} \\
&=\int_{a_{1}}^{b_{1}} \int_{a_{2}}^{b_{2}} \int_{a_{3}}^{b_{3}}\left|\omega\left(x_{1}, x_{2}, x_{3}\right) f_{1}\left(x_{1}, x_{2}, x_{3}\right) \Phi^{p-1}\left(x_{1}, x_{2}, x_{3}\right)\right| \diamond_{\alpha} x_{1} \diamond_{\alpha} x_{2} \diamond_{\alpha} x_{3} \\
& \quad+\cdots+\int_{a_{1}}^{b_{1}} \int_{a_{2}}^{b_{2}} \int_{a_{3}}^{b_{3}}\left|\omega\left(x_{1}, x_{2}, x_{3}\right) f_{m}\left(x_{1}, x_{2}, x_{3}\right) \Phi^{p-1}\left(x_{1}, x_{2}, x_{3}\right)\right| \diamond_{\alpha} x_{1} \diamond_{\alpha} x_{2} \diamond_{\alpha} x_{3} \\
& \leq \\
& \quad\left(\int_{a_{1}}^{b_{1}} \int_{a_{2}}^{b_{2}} \int_{a_{3}}^{b_{3}}\left|\omega\left(x_{1}, x_{2}, x_{3}\right)\right|\left|\Phi\left(x_{1}, x_{2}, x_{3}\right)\right|^{p} \diamond_{\alpha} x_{1} \diamond_{\alpha} x_{2} \diamond_{\alpha} x_{3}\right)^{\frac{1}{q}} \\
& \quad {\left[\sum_{i=1}^{m}\left(\int_{a_{1}}^{b_{1}} \int_{a_{2}}^{b_{2}} \int_{a_{3}}^{b_{3}}\left|\omega\left(x_{1}, x_{2}, x_{3}\right)\right|\left|f_{i}\left(x_{1}, x_{2}, x_{3}\right)\right|^{p} \diamond_{\alpha} x_{1} \diamond_{\alpha} x_{2} \diamond_{\alpha} x_{3}\right)^{\frac{1}{p}}\right] }
\end{aligned}
$$

Thus we obtain the desired inequality (25).

\section{Conclusions}

As is well known, the Hölder inequality and its various extensions play a very important role in mathematical analysis. In this paper, based on the definition of the triple DiamondAlpha integral for functions of three variables, we have presented the Hölder and reverse Hölder inequalities for the triple Diamond-Alpha integral on time scales. Moreover, we gave some new generalizations of the Hölder and reverse Hölder inequalities for the triple Diamond-Alpha integral. Finally, using the obtained results, we have obtained a new generalization of the Minkowski inequality for the triple Diamond-Alpha integral on time scales. In the future research, we will continue to explore other inequalities for the triple Diamond-Alpha integral on time scales.

\section{Acknowledgements}

This work was supported by the Fundamental Research Funds for the Central Universities (Nos. 2015ZD29 and 13ZD19) and the Higher School Science Research Funds of Hebei Province of China (No. Z2015137).

\section{Competing interests}

The author declares that there is no conflict of interests regarding the publication of this paper.

Authors' contributions

The author read and approved the final manuscript.

\section{Publisher's Note}

Springer Nature remains neutral with regard to jurisdictional claims in published maps and institutional affiliations.

Received: 12 February 2018 Accepted: 26 April 2018 Published online: 10 May 2018

\section{References}

1. Hilger, S.: Ein Maßkettenkalk ül mit Anwendung auf Zentrumsmannigfaltigkeiten. Ph.D. thesis, Universität Würzburg (1988)

2. Agarwal, R.P., Bohner, M., Peterson, A.: Inequalities on time scales: a survey. Math. Inequal. Appl. 4(4), 535-557 (2001)

3. Bohner, M., Peterson, A.: Dynamic Equations on Time Scales. Birkhäuser, Basel (2001)

4. Barić, J., Bibi, R., Bohner, M., Nosheen, A., Pečarić, J.: Jensen Inequalities on Time Scales. Theory and Applications. Element, Zagreb (2015)

5. Bohner, M., Peterson, A.: Advances in Dynamic Equations on Time Scales. Birkhäuser, Boston (2003) 
6. Hilger, S.: Analysis on measure chains-a unified approach to continuous and discrete calculus. Results Math $18(1-2), 18-56(1990)$

7. Hilger, S.: Differential and difference calculus—unified!. Nonlinear Anal. 30(5), 2683-2694 (1997)

8. Rogers, J.W.J., Sheng, Q.: Notes on the diamond- $\alpha$ dynamic derivative on time scales. J. Math. Anal. Appl. 326(1), 228-241 (2007)

9. Sheng, Q.. Fadag, M., Henderson, J., Davis, J.M.: An exploration of combined dynamic derivatives on time scales and their applications. Nonlinear Anal., Real World Appl. 7(3), 395-413 (2006)

10. Wong, F.H., Yeh, C.C., Lian, W.C.: An extension of Jensen's inequality on time scales. Adv. Dyn. Syst. Appl. 1(1), 113-120 (2006)

11. Mitrinović, D.S.: Analytic Inequalities. Springer, New York (1970)

12. Kuang, J.: Applied Inequalities. Shandong Science Press, Jinan (2003)

13. Agahi, H., Ouyang, Y., Mesiar, R., Pap, E., Štrboja, M.: Hölder and Minkowski type inequalities for pseudo-integral. Appl. Math. Comput. 217(21), 8630-8639 (2011)

14. Zhao, Y., Yan, T., Ouyang, Y.: Several inequalities for the pan-integral. Inf. Sci. 372, 625-633 (2016)

15. Tian, J., Yang, Z.-H.: Generalizations of Hu-type inequalities and their applications. J. Nonlinear Sci. Appl. 10(4), 1971-1985 (2017)

16. Tian, J.-F., Ha, M.-H.: Extensions of Hölder-type inequalities on time scales and their applications. J. Nonlinear Sci. Appl. 10(3), 937-953 (2017)

17. Tian, J.-F., Ha, M.-H.: Properties of generalized sharp Hölder's inequalities. J. Math. Inequal. 11(2), 511-525 (2017)

18. Tian, J.-F., Ha, M.-H., Wang, C.: Improvements of generalized Hölder's inequalities and their applications. J. Math. Inequal. 12(2), 459-471 (2018). https://doi.org/10.7153/jmi-2018-12-34

19. Tian, J., Ha, M.-H.: Properties and refinements of Aczél-type inequalities. J. Math. Inequal. 12(1), 175-189 (2018)

20. Tian, J., Wang, W., Cheung, W.-S.: Periodic boundary value problems for first-order impulsive difference equations with time delay. Adv. Differ. Equ. 2018, 79 (2018). https://doi.org/10.1186/s13662-018-1539-5

21. Tian, J.-F., Pedrycz, W.: New refinements of generalized Hölder's inequality and their applications. Math. Inequal. Appl. 19(3), 805-822 (2016)

22. Yang, Z.-H., Tian, J.: Monotonicity and sharp inequalities related to gamma function. J. Math. Inequal. 12(1), 1-22 (2018)

23. Yang, Z.-H., Tian, J: Optimal inequalities involving power-exponential mean, arithmetic mean and geometric mean. J. Math. Inequal. 11(4), 1169-1183 (2017)

24. Yang, Z.-H., Tian, J.: Monotonicity and inequalities for the gamma function. J. Inequal. Appl. 2017, 317 (2017)

25. Zhao, C.-J., Cheung, W.-S.: Hölder's reverse inequality and its applications. Publ. Inst. Math. 99(113), 211-216 (2016)

26. Wong, F.H., Yeh, C.C., Yu, S.L., Hong, C.H.: Young's inequality and related results on time scales. Appl. Math. Lett. 18(9), 983-988 (2005)

27. Özkan, U.M., Sarikaya, M.Z., Yildirim, H.: Extensions of certain integral inequalities on time scales. Appl. Math. Lett. 21(10), 993-1000 (2008)

28. Hardy, G.H., Littlewood, J.E., Pólya, G.: Inequalities. Cambridge University Press, Cambridge (1952)

\section{Submit your manuscript to a SpringerOpen ${ }^{\circ}$ journal and benefit from:}

- Convenient online submission

- Rigorous peer review

- Open access: articles freely available online

- High visibility within the field

- Retaining the copyright to your article

Submit your next manuscript at $\boldsymbol{\nabla}$ springeropen.com 